\title{
Extraction of Grayscale Brain Tumor in Magnetic Resonance Image
}

\author{
Yamini Sharma ${ }^{1}$, Yogesh K. Meghrajani ${ }^{2}$ \\ Department of Electronics and Communication, Dharmsinh Desai University, Nadiad, Gujarat, India ${ }^{1}$ \\ Associate Professor, Dept of Electronics and Communication, Dharmsinh Desai University, Nadiad, Gujarat, India ${ }^{2}$
}

\begin{abstract}
Magnetic resonance imaging (MRI) has great contribution in diagnosis and treatment of brain tumor. Segmentation of grayscale tumor or low intensity tumor is the most important and challenging task. In this paper, we propose a method to extract grayscale tumor from MRI image. Due to low intensity profile of input image, global contrast enhancement method is applied as a pre-processing step. This enhanced grayscale image is converted into binary image. Further, algorithm segments largest connected region using mathematical morphological and segmentation methods. At last, tumor region is extracted using resultant image and input MRI image. Experimental results show the proposed method effectively extracts a grayscale brain tumor from MRI images.
\end{abstract}

Keywords: MRI image, grayscale tumor, mathematical morphology and segmentation.

\section{INTRODUCTION}

Brain tumor is one of the most widespread brain diseases that has affected and devastated many lives. As per International Agency for Research on Cancer (IARC), approximately, more than 126000 people are diagnosed for brain tumor every year around the world, with more than 97000 mortality rate [1]. Statistics shows low survival rate of brain tumor patients, despite of consistent efforts to overcome the problem of brain tumor. Brain tumor; briefly described, as under certain conditions, brain cells grow and multiply uncontrollably due of some reasons. Mechanism that control normal cells is unable to regulate the growth of the brain cells. World Health Organization (WHO) classified brain tumor into 120 types.

There are two categories of tumor based on the originprimary and secondary brain tumors. Primary brain tumors are originated from the brain and are named for the cell types from which they originated. They can be benign (non-cancerous) and malignant (cancerous). Benign tumors grow slowly and do not spread elsewhere or invade the surrounding tissues. Malignant tumors are more aggressive that grow more quickly and spread to other tissues.

Secondary brain tumors originate from another part of the body. These tumors consist of cancer cells somewhere else in the body that spread to the brain. The most common cause of secondary brain tumors are: lung cancer, breast cancer, melanoma, kidney cancer, bladder cancer, etc.[2]. Magnetic resonance imaging (MRI) is the most preferred imaging technique in radiology because MRI enables internal structures to visualize in detail. This makes MRI suitable for providing better quality images for the brain, the muscles, the heart and cancerous tissues compared with other medical imaging techniques, such as computed tomography (CT) or X-rays [3].
Many researchers have proposed techniques to extract brain tumor from MRI image. Kharrat et al. [7] used the $\mathrm{K}$-means algorithm to extract the tumor considering high intensity of brain tumor. Shape of the extracted tumor is distorted in their result by using K-means. Gadpayle et al. [8] used morphological operators to separate the tumor part of the image and assumed tumor portion has the highest intensity than any other regions of the image. However, this assumption does not give satisfactory result for low-level intensity of tumor. Bauer et al. [4] utilized atlas-based segmentation of brain tumor using a Markov random field-based tumor growth model and non-rigid registration on MRI images having grayscale or low intensity tumor. Nevertheless, their method selects seed point manually and the fine details of the shape of extracted tumor not clearly visualized.

In this paper, we propose a method for extracting brain tumor of low intensity from grayscale MRI image. Initially, image is cropped using vertical as well as horizontal profile and histogram equalization is used for intensity enhancement of cropped grayscale image. Afterward, global thresholding is applied for converting cropped histogram equalized MRI image into binary image. Morphological opening operation is applied on this binary image. Further, segmentation of connected component algorithm is applied on the resultant image of the opening operation. From these connected component regions, largest connected component region is selected. Finally, tumor region is extracted by multiplying resultant image with input MRI image.

This paper is organized as follows. In section II, we describe methodology. The experimental results are presented in section III and in section IV, we conclude this paper. 


\section{Methodology}

Brain MRI image of grayscale or low intensity tumor is obtained as input image. Furthermore, image is cropped, enhanced, mathematical morphological operations likeopening is applied and then finally tumor is segmented using extraction of connected component algorithm.

\section{A. Image cropping}

Grayscale brain tumor MRI image is cropped by applying vertical horizontal profile. Image cropping is performed first to improve image enhancement, otherwise background region affects intensity enhancement.

\section{B. Image intensity enhancement}

Cropped brain MRI image is enhanced using intensity enhancement techniques like histogram equalization. Histogram equalization is applied to enhance the image uniform distribution of the pixel intensity and to boost the visualization of the cropped MRI image consisting of low intensity brain tumor.

\section{Segmentation of tumor}

For segmenting tumor- thresholding, mathematical morphological operation and segmentation method are performed on histogram-equalized image. Minimum value in histogram-equalized image is set to zero. Intensity of histogram-equalized image from one to 127 is set to one and intensity above 127 is set to zero. This way thresholding of histogram equalized image is performed by keeping brain segment background as black. The reason of keeping brain segment background as black (minimum intensity) is so that it (background) will not affect the tumor portion of the threshold image. Expression of global thresholding is given by (1), where G $(\mathrm{x}, \mathrm{y})$ is output binary image, $\mathrm{T}$ is threshold value and $\mathrm{F}(\mathrm{x}, \mathrm{y})$ is input histogram equalized image.

$$
\mathrm{G}(\mathrm{x}, \mathrm{y})= \begin{cases}0 & \text { if } \mathrm{F}(\mathrm{x}, \mathrm{y})>T \\ 1 & \text { if } \mathrm{F}(\mathrm{x}, \mathrm{y}) \leq \mathrm{T}\end{cases}
$$

Morphological opening is applied on binary image. Structuring element (SE) of square shape and size 3 is used. Expression of morphological opening is given by (2), where $\mathrm{A}$ is the input image given to the opening operation and B is the SE [5].

$$
\mathrm{A} \circ \mathrm{B}=(\mathrm{A} \ominus \mathrm{B}) \oplus \mathrm{B}
$$

Extraction of connected components algorithm is applied on the resultant image of opening operation. As a result, all connected components are obtained and the largest connected component region is selected from all connected regions presuming tumor is the largest region. Finally, mathematical multiplication operation is applied on original input image and resultant image obtained after extraction of largest connected component among all connected components. As a result, tumor region similar to original image is obtained. The flowchart of the proposed method is as shown in Fig. 1.

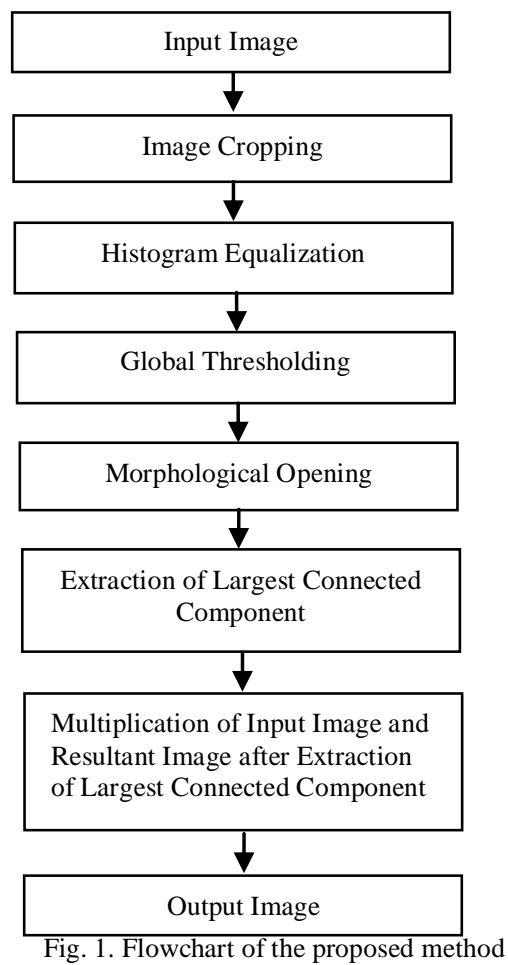

III.EXPERIMENTAL RESULTS

Stepwise results of the proposed method are shown in Fig. 2 , it comprises of different images : 1. Input image, 2. Cropped image, 3. Histogram-equalized image, 4. Global threshold image, 5. Image after applying morphological opening operation, 6. Image showing largest pixel connected region. At the end of process, final output is obtained. The complete flow of the proposed approach is visualized from the figure.

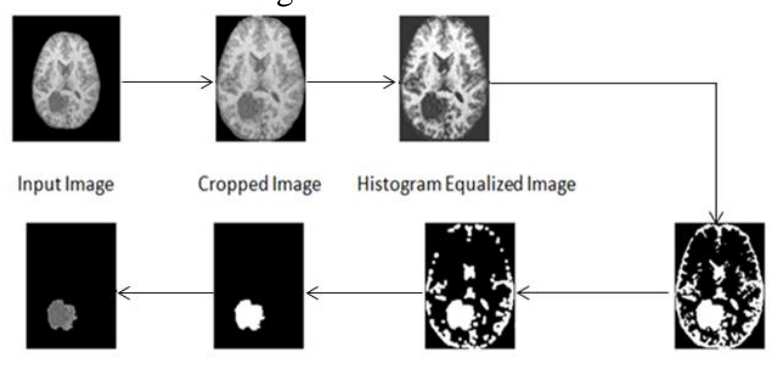

Final Output Image Extraction of largest region Result of Openin Threshold Image

Fig.2. Stepwise results of the proposed method

A. Comparison with related grayscale tumor segmentation method and the proposed scheme

\section{IV.TABLE I}

COMPARISON OF RESULTS

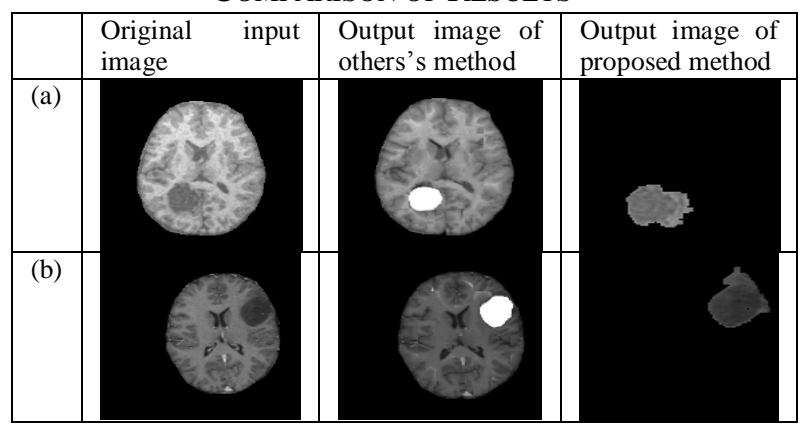


In Table I, the results of Bauer et al. [4] show that the fine details of the shape of tumor not clearly visualized after segmenting it and limitation of their method is that seed point is selected manually. The result of our proposed method shows fine details of the shape of extracted tumor and our method is independent of seed point. However, in Table I (b), due to small segment of background is connected with tumor and its intensity is analogous to the tumor,it appears in resultant image.

\section{A. Performance analysis}

In addition to subjective analysis, for objective or mathematical analysis, we calculate peak signal to noise ratio (PSNR) [6] given by (3),

$$
\text { PSNR }=10 \log _{10}\left(\frac{\mathrm{R}^{2}}{\mathrm{MSE}}\right)
$$

Where, for 8-bit unsigned integer data type (as in our case), $\mathrm{R}$ is 255 andMSE (mean square error) [5], is given by (4), where $\mathrm{F}(\mathrm{x}, \mathrm{y})$ is original image and $\hat{F}(x, y)$ is reconstructed image,

$$
M S E=\left[\frac{1}{M N}\right] \sum_{x=0}^{M-1} \sum_{y=0}^{N-1}[F(x, y)-\hat{F}(x, y)]^{2}
$$

PSNR computes the peak signal-to-noise ratio, in decibels, between two images. This ratio is used as a quality measurement between the original and a reconstructed image. Here, output images of extracted tumor region using proposed method for input images (a) and (b) in Table I, are considered as reconstructed images, whereas manually cropped tumor region of input cropped images (using vertical and horizontal profile) are considered as original images. Measured PSNR values are as shown in Table II. Input image-1 shows less PSNR value due to complicated manual cropping of fine details of input image.

TABLE II

PERFORMANCE ANALYSIS

\begin{tabular}{|l|l|l|}
\hline & \multicolumn{1}{|c|}{ Image } & \multicolumn{1}{|c|}{ PSNR(dB) } \\
\hline 1 & Input Image-1 & 23.6 \\
\hline 2 & Input Image-2 & 30.28 \\
\hline
\end{tabular}

\section{Conclusion}

The proposed method presents segmentation of grayscale brain tumor using image enhancement techniques, mathematical morphological and other segmentation techniques. Due to low intensity of tumor region, input image intensity is enhanced using histogram equalization, which enhances global contrast of the image. As histogram equalization utilizes entire image to enhance global contrast, excess background region affects intensity enhancement. Hence, input image is cropped using horizontal and vertical profile before applying histogram equalization. Further, global thresholding, mathematical morphological opening, and largest connected component algorithm segment the tumor region assuming tumor is the largest region. Notably, algorithm extract tumor region independently.

\section{REFERENCES}

[1] J. Ferlay, H.R. Shin, F. Bray, D. Forman, C. Mathers, and D.M. Parkin, GLOBOCAN 2008 v2.0, CancerIncidence and Mortality Worldwide, International Agency for Research on Cancer, Lyon, France, 2010.http://globocan.iarc.fr, Accessed on: August 10, 2014.

[2] D.N. Louis, H. Ohgaki, O.D. Wiestler, and W.K. Cavenee (Eds.), "WHO classification of tumors of the central nervous system," International Agency for Research on Cancer (IARC), Lyon, France, 2007

[3] Squire, L.F., Novelline, and R.A., Squire's Fundamentals of Radiology, Harvard University Press, ISBN 0674012798, 2014.

[4] S. Bauer, C. Seiler, T. Bardyn, P. Buechler, and M. Reyes," Atlasbased segmentation of brain tumor images using a Markov random field-based tumor growth model and non-rigid registration," Annual International Conference of the IEEE Engineering in Medicine and Biology Society, 2010.

[5] R. Gonzalez, and R. Woods, Digital Image Processing, Third edition, NJ: Prentice Hall

[6] Computation of peak signal-to-noise ratio (PSNR) between images, http://www.mathworks.in/help/vision/ref/psnr.html.Accessed on: October 06, 2014.

[7] A. Kharrat, M.B. Messaoud, N. Benamrane, and M. Abid, "Detection of brain tumor in medical images," IEEE 3rd International Conference on Signals, Circuits and Systems, 2009.

[8] P. Gadpayle, and P.S.Mahajani,"Detection and classification of brain tumor inMRI images," International Journal of Emerging Trends in Electrical and Electronics, vol. 5, no. 1, 2013.

\section{BIOGRAPHIES}

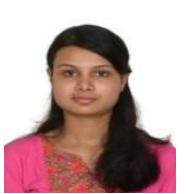

Yamini Sharma received the Bachelor of Engineering Degree in Electronics and communicationEngineering in 2013 from Gujarat Technological University, Ahmedabad, Gujarat, India. She is pursuing Master of Technology in Electronics and communication Systems from Dharmsinh Desai University, Nadiad, Gujarat, India. Her area of interest is, Image processing, and signal processing.

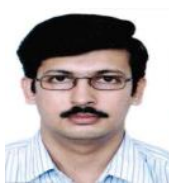

Yogesh K. Meghrajani obtained his Master's degree in Communication System Engineering from Gujarat University, Ahmedabad, India, and is currently pursuing Ph. D. degree. In 2002, he joined as a lecturer in Electronics \& Communication department, Dharmsinh Desai University, Nadiad, Gujarat, India, where he is currently working as an Associate Professor since 2007. His research areas include cryptography, image processing, and embedded systems. 